\title{
Genetic variation in host plant phenology affects microbial assemblages at longitudinal niches of the seedling root
}

\author{
Yi Zhou ${ }^{1}$, Sheikh Rabbi ${ }^{2}$, Iain Young ${ }^{2}$, David Fuentes ${ }^{2}$, Andrew Merchant $^{2}$, Brent Kaiser ${ }^{2}$, \\ Yongle $\mathrm{Li}^{1}$, Jishun $\mathrm{Li}^{3}$, Yanli $\mathrm{Wei}^{3}$, and Matthew D. Denton ${ }^{1}$ \\ ${ }^{1}$ The University of Adelaide School of Agriculture Food \& Wine \\ ${ }^{2}$ The University of Sydney Faculty of Science \\ ${ }^{3}$ Qilu University of Technology
}

February 21, 2022

\begin{abstract}
The root tip of a plant is highly sensitive to environmental cues and exerts sensory, chemical and movement responses and has even been likened to an animal's brain. Yet, the assemblage of microbes at the root tip, the control of their diversity, the nature of their recruitment to that particular volume, and their roles in plant phenotypic function, remain poorly understood. This study investigated longitudinal niche differentiation of the root-associated microbiome in chickpeas ( Cicer arietinum L.) and its interactions with both diverse soil types and host plants with genetic variation in phenology, from the exterior to the interior of the root. Compared with late flowering (LF) genotypes, endophyte microbiomes at the apical zone of the early flowering (EF) host were characterised by greater diversity, higher compositional similarity to the basal zone, and closely inhabiting Rhizobacter and Methylotenera across soils. Additionally, EF genotypes secreted a specific composition of metabolites from the apical zone, with more carboxylates and amino acids (especially propionic acid and benzoic acid) than the LF plant. Our findings demonstrate that longitudinal differentiation within a seedling root is an essential feature shaping the root microbiome and indicative of genetic variation in phenology of host plants.
\end{abstract}

\section{Introduction}

In his book "The Power of Movement in Plants" (Darwin, 1897) Charles Darwin postulated the "RootBrain" hypothesis where he identified similarities between the plant root tip and the simple brain of lower metazoans, including sensory perception of external stimuli and physiological responses. The root apex is currently attracting both intensive and extensive attention related to plant signalling and development, as it is the zone for root elongation, cell division, and hormone crosstalk, triggered by sensing diverse physical and chemical gradients and/or thresholds such as gravity, moisture, mechanical resistance, light, and inorganic nutrients (Benizri et al., 2001; Baluška et al., 2010).

The apical zone of a root plays a greater role than the basal zone in coordinating the formation of rootassociated microbiota. Firstly, a plant can manipulate the soil microbiome by secreting root exudates as substrates to direct the microbial life close to the root (Sasse et al., 2017; Zhou et al., 2020). Most of these exudates arise from the zone just behind the root tip, due to the absence of an apoplastic barrier (i.e., Casparian strip, suberin or schlerenchyma) which favours passive diffusion of compounds through the plasma membrane (Canarini et al., 2019). Additionally, the root tip is the first tissue contacting soil - the 'seed bank' of any plant microbiome. Soil microbes may penetrate into the central cell layers of the root tip, prior to differentiation of the endodermis, which later forms a thick-walled boundary in the mature zone (ReinholdHurek and Hurek, 1998). Changes in the patterns of microbiome assemblages between the root tip and the basal root within the same root system were found in Avena fatua (DeAngelis et al., 2009), Brachypodium 
distachyon (Kawasaki et al., 2016), maize (Rüger et al., 2021), wheat, and rice (Kawasaki et al., 2021b; Kawasaki et al., 2021a). However, the previous evaluation of root microbiomes at longitudinal niches has only considered a single soil type, host genotype, or rhizocompartment (rhizosphere or endosphere), and failed to provide deeper insights into the complexity of these interactions in structuring root microbiota.

The specific length of the root region is generally defined as apical or basal root for microbiome analysis (DeAngelis et al., 2009; Kawasaki et al., 2016; Kawasaki et al., 2021b; Kawasaki et al., 2021a; Rüger et al., 2021). This length-based definition might lead to different soil residence times for the apical or basal root segments when growing under different environments, because the elongation rate of plant root varied under different soil types and for different plant genotypes. Microbiome assembly in root is rapid (e.g., $24 \mathrm{~h}$, Edwards et al., 2015) and sensitive to the soil residence period (Dombrowski et al., 2017), so root segments with the same soil residence time should be sampled to compare their microbiomes under different soils or for different host genotypes, which has not been considered in previous studies.

Plant phenology is an important trait for crop domestication and improvement and has strong interactions with the root-associated microbiome. Root microbiomes of the perennial plant Arabis alpinewas influenced by soil residence periods, but was indistinguishable between the nonflowering wild type and the early flowering mutant (Dombrowski et al., 2017). For annual rice genotypes, both the developmental rate and soil residence time determined the microbiota assembly through the life cycle (Edwards et al., 2018). A recent mode showed that the flowering time of Arabidopsis was driven by rhizosphere microbes that were modulated by root exudates ( $\mathrm{Lu}$ et al., 2018). As there was spatial variation of root exudates within a root system, microbiome acquisition along the longitudinal root axis after a short soil-residence time might indicate the genetic variation of host plant phenology.

Chickpea (Cicer arietinum L.) originated from a small area in southeast Turkey (Von Wettberg et al., 2018). The selection in chickpea's domestication focuses on photoperiod-responsive and vernalization-insensitive genotypes to ensure the life cycle competed before the summer heat and drought (Abbo et al., 2002). The genomic analysis revealed that an 11-bp deletion in the early flower geneELF3 was associated with early flowering among chickpea cultivars (Ridge et al., 2017). In addition, chickpea is characterised by late lateral root initialisation, acidic root exudates (Wang et al., 2016; Zhou et al., 2020), and specific symbiosis with Mesorhizobium ciceri (Greenlon et al., 2019). The objectives of our study were to (1) investigate the mechanisms that structure the root microbiome and modulate root exudation associated with genetic variation in flowering phenology, and (2) identify longitudinal niche differentiation of the root-associated microbiome, and its interactions with soil type, genotype, and rhizocompartments from the exterior to the interior of the root.

\section{Materials and Methods}

\section{Experiment 1: rhizotron growing systems and samples collection for microbial analysis}

A rhizotron system $(30 \mathrm{~cm}$ long $\times 18 \mathrm{~cm}$ wide $\times 2 \mathrm{~cm}$ thick) with a removable transparent acrylic side was designed for the current experiments. Rhizotrons were covered with aluminium foil to simulate dark conditions for root growth, and angled at 45 degrees, with the transparent side as the base, to enable visualisation of roots under the transparent surface. Five soil types and three chickpea genotypes with 4 replicates were examined in this rhizotron system. Five soils were collected from the cropping regions of South Australia, Australia, and marked as Soil-A (-34.511, 138.697), Soil-B (-34.509, 138.667) and Soil-C $(-34.537,138.690)$, Soil-D $(-34.967,138.633)$ and Soil-E $(-34.966,138.631)$. All the soils used here were from typical Mediterranean dryland farming systems with 3 to 4 years of cereal crops (wheat or barley) rotated with one season legume (lentil, field pea or faba bean). To limit the excessive presence of Mesorhizobium ciceri (the symbiotic rhizobia of chickpea), soils were chosen where no chickpea was planted in the last decade. The soils differed in chemical and physical properties (Table S1), which enabled the evaluation of stochastic or deterministic processes of host plants in assembling microbiome at different niches. Soil physiochemical properties including total $\mathrm{N}, \mathrm{C}, \mathrm{P}, \mathrm{K}, \mathrm{Ca}, \mathrm{Zn}, \mathrm{Mg}, \mathrm{Mn}$ and $\mathrm{Cu}, \mathrm{pH}, \mathrm{EC}$, and texture were analysed using the protocol from Rayment \& Lyons (2011), and the results are presented in Table S1. Chickpea cultivars 
Genesis090, PBA Slasher and Sonali were used. Previous evaluation across multiple environments with diverse photoperiod, seasonal rainfall, and cropping management during chickpea growth (Sadras et al., 2016; Kaloki et al., 2019) have demonstrated that Sonali (genotype EF) required less thermal time to flowering compared with the late flowering genotypes Genesis090 (genotype LF1) and Slasher (genotype LF2). It has been confirmed that an 11-bp deletion in chickpea early flowering 1 (Efl1) locus was associated with early flowering performance (Ridge et al., 2017). We analysed the structure of Efl1 gene by sequencing the whole genomes of the three cultivars in the present study. DNA sequencing and mapping were described in (Li et al., 2017). Briefly, genomic DNA of Genesis090, PBA Slasher, and Sonali was extracted from young leaf tissue using Qiagen DNeasy Plant Mini Kit. Pair-end reads (100 bp) of each genotype were sequenced using Illumina HiSeq 2000 platform. Sequencing reads were filtered, trimmed, and mapped to the reference genome 2.6 .3 (http://cicer.info) using SOAP2. Bam files were filtered out for reads with more than 3 mismatches resulting in $\sim 10 \mathrm{x}$ coverage of each genome. Bam files were imported into the software Integrated Genome Viewer (IGV) for visual inspection of the Efl1 gene.

Chickpea seeds were surface-sterilized by soaking in $10 \%$ calcium hypochlorite for $10 \mathrm{~min}$, and were then washed with sterile water three times, soaked in $70 \%$ ethanol for $20 \mathrm{~s}$, and then washed another three times in sterile water. Surface-sterilised seeds were germinated in a petri dish with autoclaved sand and water until the root emerged to a length of $0.5 \mathrm{~mm}$. Two germinated seeds were transplanted to $2 \mathrm{~cm}$ depth in each sterilised rhizotron that had been filled with soil at a consistent bulk density of $1.38 \mathrm{Mg} \mathrm{m}^{-3}$. Rhizotrons without plants were used as the bulk soil control.

Plants were grown in a controlled environment room $\left(800 \mu \mathrm{mol} \mathrm{m}{ }^{-2} \mathrm{~s}^{-1}\right.$ light for 12 hours at $20{ }^{\circ} \mathrm{C}$ and 12 hours of darkness at $10{ }^{\circ} \mathrm{C}$ ), and watered to field capacity every two days by weighing using autoclaved sterilised water. The point of observable root tips was marked each day. At 16 days after planting (DAP), roots with rhizosphere soil were harvested. Root segments between the markers of 1 to 2 DAP and 15 to 16 DAP were cut off with approximately $1.0 \mathrm{~mm}$ of soil adhering around the root; these were regarded as the apical and basal zones, respectively. Root segments from two plants in each rhizotron were processed for microbial community analysis. Rhizosphere and endosphere samples were isolated from root sections using a method modified from Bulgarelli et al. (2012). The sampled root segments with attached soil were transferred into $15 \mathrm{~mL}$ sterile PBS buffer in a $50 \mathrm{~mL}$ Falcon tube, which was placed on an orbital shaker for 20 min and rotated at $180 \mathrm{rpm}$. Roots were collected using sterilised tweezers for further processing. The rest of the soil suspension was centrifuged at $180 \mathrm{rpm}$ for $20 \mathrm{~min}$. After removing the supernatant, the pellet was defined as rhizosphere soil. Roots were washed again on the shaker for $20 \mathrm{~min}$ at $180 \mathrm{rpm}$, and then transferred into a new $50 \mathrm{~mL}$ Falcon tube with $40 \mathrm{~mL}$ sterile PBS buffer. The roots in the Falcon tube were sonicated for 10 cycles of $30 \mathrm{~s}$ at $43 \mathrm{kHz}$ and $120 \mathrm{~W}$ (model 160HD, Soniclean, Australia) with a $30 \mathrm{~s}$ break. The microbiome extracted from the sonicated root was considered to be from the endosphere. The rhizosphere and endosphere samples were stored in a $-80{ }^{\circ} \mathrm{C}$ freezer.

The total DNA of the rhizosphere soil, cleaned root tissue, and bulk soil was extracted using a PowerSoil DNA isolation kit (Mo Bio, Carlsbad, CA, USA) based on the manufacturer's instructions. Afterwards, the V3-V4 region of $16 \mathrm{~S}$ rRNA genes was amplified using the forward primer 341F (CCTAYGGGRBGCASCAG) and reverse primer 806R (GGACTACNNGGGTATCTAAT). The PCR products were sequenced by Australian Genome Research Facility on an Illumina MiSeq platform with 300 bp paired-end reads following the manufacturer's instructions.

Quantitative PCR (qPCR) was performed to determine the quantity of bacterial DNA in the samples, using the Femto ${ }^{\mathrm{TM}}$ Bacterial DNA Quantification Kit (Zymo Research, USA) following the manufacturer's instructions. The universal bacterial $16 \mathrm{~S}$ rRNA gene was amplified on the CFX connect Real-Time PCR detection system (Bio-Rad, USA), and gene copy numbers were estimated using E. coli strain JM109 as an internal standard. Three technical replicates per sample were analysed. Data were calculated as copy number per gram of the soil or root tissue used for DNA extraction.

Experiment 2: root exudates collection and analysis 
For the root exudate collection, three chickpea genotypes were planted in the rhizotrons as described in Experiment 1 with 4 replicates. Only Soil-C was used, but sterilised by gamma radiation, as soil microbes can degrade the root exudates rapidly. The growing condition and management were the same as Experiment 1. Sample preparation for root exudate analysis was based on a method modified from Wouterlood et al. (2004). The attached soil on sampled apical and basal segments of roots were transferred into $50 \mathrm{~mL}$ Falcon tube with $10 \mathrm{~mL}$ deionized water, and then microwaved by a $700 \mathrm{~W}$ microwave oven for $10 \mathrm{~s}$ to prevent metabolic activities. The sample was washed on an orbital shaker for $30 \mathrm{~min}$ at $180 \mathrm{rpm}$ and the root was then discarded. The soil suspension was filtered through Whatman no. 42 filter paper. The filtered solution was transferred to a syringe filter $(0.1 \mathrm{um})$, and then stored at $-80{ }^{\circ} \mathrm{C}$ for further analysis. The soil collected was dried at $105{ }^{\circ} \mathrm{C}$ to measure soil dry weight.

Root exudates were analysed for carbohydrates and amino acids at the Centre for Carbon, Water and Food in The University of Sydney, following the method in Conselvan et al. (2018). Carbohydrate and sugar analysis were measured by gas chromatography (GC). The analysis was carried out on an Agilent 6890 Gas Chromatograph with QQQ 7000 Mass selective detector with a HP5 column $(0.25 \mathrm{~mm}$ i.d., $30 \mathrm{~m}, 0.25 \mathrm{~mm}$ film thickness; Agilent Technologies, Santa Clara, United States). Split injection was made at $300{ }^{\circ} \mathrm{C}$ with an initial oven temperature program of $60{ }^{\circ} \mathrm{C}$ for $2 \mathrm{~min}$, ramping to $300{ }^{\circ} \mathrm{C}$ at a rate of $10{ }^{\circ} \mathrm{C} \mathrm{min}{ }^{-1}$ and maintained for $10 \mathrm{~min}$. Column flow rate was maintained at $1.5 \mathrm{~mL} \mathrm{~min}{ }^{-1}$. Mass Hunter software (version B.07.01, Agilent Technologies) was used for peak integration. For amino acid analysis, LC-MS analysis of the extract was carried out on a 1290 Infinity LC system coupled to a 6520 QTOF Mass selective detector (Agilent Technologies, Santa Clara, United States). A 3.5 uL sample was injected into a Zorbax SB-C18 column $(2.1 \times 150 \mathrm{~mm}, 3.5 \mu \mathrm{m})$ and separation was achieved by gradient elution with water and methanol. The QTOF was tuned to operate at the low mass range $<1700$ AMU and data acquisition was performed in scan mode $(60-1000 \mathrm{~m} / \mathrm{z})$ and ionization was set to positive ion mode. LC-MS results were identified based on their retention times relative to standards as well as their formula mass. Peaks were integrated and their relative quantities were calculated using the Mass Hunter software. The measured root exudate compounds were estimated relative to the amount of dry soil and growing period $(1 \mathrm{~d}$ for apical root and $15 \mathrm{~d}$ for basal root).

\section{Experiment 3: rhizosphere soil architecture analysis}

To study soil architecture of the rhizosphere using micro-CT scan, three chickpea genotypes were planted in the PVC tubes $(5 \mathrm{~cm}$ diameter $\times 30 \mathrm{~cm}$ height to fit the micro-CT scanner) using the same 5 soils with the same soil bulk density and growing conditions to Experiment 1. At 16 DAP, a period when no roots were observed at the bottom of every PVC tube, plant shoots were cut off, and the tube with soil and roots was used for micro-CT scanning. The PVC tubes with chickpea roots were scanned using X-ray microtomography (Phoenix|X-ray, GE Sensing \& Inspection, Wunstorf, Germany) at $160 \mathrm{kV}$ and $200 \mu \mathrm{A}$ with a voxel resolution of $\sim 16 \mu \mathrm{m}$ to extract the $3 \mathrm{D}$ root system and measure the pore architecture of the rhizosphere and bulk soil (Rabbi et al., 2018; Rabbi et al., 2021). The top and bottom $1 \mathrm{~cm}$-long roots were considered as the basal and apical roots, respectively. The roots were extracted from 3D image slices using ROOT1 (Flavel et al., 2017) plug-in for Fiji (Schindelin et al., 2012). The segmentation of soils in pores and solids were performed with the image thresholding algorithm in Fiji. The pores within 0 to $1 \mathrm{~mm}$ (defined as rhizosphere) and from 1 to $2 \mathrm{~mm}$ (defined as bulk soil) from the root surface were extracted and analysed using RHIZOSPHERE macro (Rabbi et al., 2018) developed for Fiji.

\section{Experiment 4: chickpea symbiotic rhizobia identification}

To identify any naturalised Mesorhizobium able to nodulate chickpea from the soil, the same three chickpea genotypes were grown in the pots $(20 \mathrm{~cm}$ diameter $\times 20 \mathrm{~cm}$ tall $)$ filled with the same 5 soils used in Experiment 1 with 4 replicates. The growing environment and management were same to Experiment 1. Nodules were harvested at 6 weeks after sowing, and washed by sonicator as described above. DNA was extracted from the clean nodules, amplified and sequenced using the same process as the 16S rRNA gene amplicon sequencing. 


\section{Bioinformatics}

All the sequenced samples, including bulk soil, rhizosphere, endosphere and nodule tissue, were combined for bioinformatics using the Quantitative Insights Into Microbial Ecology 2 (QIIME2) platform (Bolyen et al., 2019). Firstly, raw sequences were demultiplexed by the "q2-demux" plugin, and then trimmed and quality controlled using "q2-cutadapt" plugin, followed by denoising with DADA2 ("q2-dada2" plugin, Callahan et al., 2016) to generate an amplicon sequence variants (ASVs) table. The taxonomic annotation of ASVs was conducted by "q2-feature-classifier" plugin based on the SILVA reference database (vs. 132, Quast et al., 2012). Low abundance ASVs (ASVs with less than 5\% chance appeared in all the samples) and organellar ASVs (ASVs assigned to chloroplast or mitochondria) were removed. The raw sequenced data from the Illumina MiSeq platform are available at National Center for Biotechnology Information (NCBI), Sequence Read Archive (https://www.ncbi.nlm.nih.gov/sra) with the accession number PRJNA595445.

\section{Statistics}

As the sequenced library size was variable among samples, ASV count was normalised by the Trimmed Mean of $\mathrm{M}$ values method in the edgeR package in $\mathrm{R}$ (Robinson et al., 2010). Bray-Curtis distance was calculated between samples to indicate the differentiation of microbiome composition. Based on Bray-Curtis distance, non-metric multidimensional scaling (NMDS) plot was generated to visualize microbial community from the Vegan Package (Oksanen et al., 2007). Permutational multivariate analyses of variance (PERMANOVA) with maximum 999 permutations (adonis function from Vegan package) was conducted to test the effects of soil type, chickpea genotype, rhizocompartment, root segment, and their interactions on microbiome composition, and root segment and rhizocompartment were nested in soil and genotype. Shannon index of samples was estimated using iNEXT in R package (Hsieh et al., 2016). PERMANOVA and unconstrained principal coordinate analyses (PCoA) using Bray-Curtis distance were also conducted to analyse the composition of root exudate compounds. The Statistical Analysis of Metagenomic Profiles (STAMP) package was used to determine statistical differences of taxonomic groups between experimental treatments with BenjaminiHochberg FDR method to correct for the $\mathrm{P}$ value (Parks et al., 2014). For the variables of Bray-Curtis distance, Shannon index, CSR, bacteria number, and root exudates, General Linear Model was applied to test the effects of experimental factors and interactions using Minitab (Minitab Inc., State College, PA, USA). Normal distribution of each of the variables had been checked. Tukey HSD post-hoc pairwise comparison test was performed to compare the treatments.

\section{Results}

This study examined the interactions of root longitudinal segments (apical and basal zones), rhizocompartment (rhizosphere and endosphere), chickpea genotype, and soil type on the assemblage of the prokaryotic microbiome by sequencing $16 \mathrm{~S}$ rRNA genes. We used three chickpea genotypes, including one early flowering (EF) and two late flowering (LF1 and LF2), based on previous long-term and multi-environmental field evaluations. Genomic analysis confirmed that genotype EF carried a mutated form of the gene Efl1 with 11-bp deletion compared with genotype LF1 and LF2 (Fig. S1). The 11-bp deletion of Efl1 was associated with early flowering in the global chickpea germplasm (Ridge et al., 2017).

Five soils were collected from rainfed-cropping lands with contrasting physical, chemical and biological properties (Table S1). Using the rhizotron system with a removable transparent side, root longitudinal segments of apical zone and basal zone in the same chickpea plant were sampled, based on the rapid establishment of root microbiome reported previously (DeAngelis et al., 2009; Edwards et al., 2015). Each of the sampled longitudinal zone had the same root-soil contact period (1 day) from different soils and genotypes. We measured the length of sampled apical roots or basal roots under different soil types and genotypes, and found the interaction between soil type and genotype was significant on sampled root length (Fig. S2). As root elongation rate differs among genotypes and soil types, our rhizotron system can ensure that the sampled apical / basal zone was at the same soil residence period across different treatments.

The composition and diversity of the root-associated microbial community 
We analysed all the experimental factors and their interactions on microbial community composition. Permutational multivariate analyses of variance (PERMANOVA) showed that rhizocompartment and soil type explained the largest percentage of data variation, with $16 \%$ and $18 \%$, respectively $(\mathrm{P}<0.01$, Table 1$)$. Therefore, microbiome structure in the rhizosphere and endosphere were analysed individually.

For rhizosphere microbiome composition, soil type accounted for most of the variation (about $80 \%, \mathrm{P}<0.01$ based on PERMANOVA, Table 1), although the effects of root segment, genotype and their interactions were all significant $(\mathrm{P}<0.01$, Table 1). The biplot of non-metric multidimensional scaling (NMDS) based on Bray-Curtis distance showed that the samples clustered by soil type, and there was a high overlap between Soil-A and Soil-C (Fig. 1a). By contrast, the effects of root segment and host genotype were greater than soil type in determining the endosphere microbiome (11\%-24\% vs $6 \%$ PERMANOVA test, Table 1). In the NMDS biplot exploring root endophyte profiles, communities from the root apical and basal zone were clearly separated based on NMDS1, independently of different soil types (Fig. 1b). Interestingly, endophyte composition in apical and basal root of the genotype EF overlapped considerably in the biplot (Fig. 1b). Consequently, we calculated the Bray-Curtis distance of microbiota composition between apical and basal root within each treatment and confirmed that this distance for the EF genotype was only half of the LF genotypes (LF1 and LF2) in the endosphere ( $\mathrm{P}<0.01$, Table 1 and Fig. 1c). In addition, the variability of endophytic colonization across different soils was more stable in apical roots of all the genotypes, and in the $\mathrm{EF}$ basal root than other niches, as indicated by the shorter Bray-Curtis distance $(\mathrm{P}<0.05$, Table 1 and Fig. 1d).

At the phylum level, we analysed the taxonomic composition of the microbiome in each of the studied niches (Fig. 2). Proteobacteria and Actinobacteria were the dominant phyla in all the samples, accounting for $47 \%$ - $93 \%$ of the microbial consortia. A compensatory trend was observed in which the relative abundance of Proteobacteria increased, but Actinobacteria decreased from rhizosphere to endosphere, as well as from apical root to basal root, except for the EF genotype's basal root tissue, which harboured an unaltered phylum composition relative to its apical zone, with less Proteobacteria but more Actinobacteria than the other two LF genotypes (FDR-adjusted $\mathrm{P}<0.01$ ).

Shannon index was used to estimate alpha diversity of the microbial community in each of the sampled niches. The magnitude of rhizocompartment effects on Shannon index was the greatest, indicated by the largest $\mathrm{F}$ value $(\mathrm{P}<0.01$, Table 1$)$, and on average Shannon index was about 10 times higher in rhizosphere than endosphere samples (Fig. 3a and b). In the rhizosphere, the genotypic variation in the Shannon index was strongly dependent on the soil type and the longitudinal root fraction (Fig. 3a). While in the endosphere microbiome, the diversity of the EF genotype was greater than the two LF genotypes, and this observation was consistent in apical roots and basal roots in all soil types (Fig. 3b).

\section{Core microbiota at root niches of host genotypes}

To identify the core microbiota within a niche of host genotype, we first established a Rank-Abundance curve of the detected ASVs for each sample and considered the ASVs with relative abundance more than $90 \%$ of the most abundant ASV, to be dominant ASVs (dASVs). The dASVs appearing in all the five soils and four replicates were used as the core ASVs of the niche (Fig. S3). In the rhizosphere, there were 2 core ASVs shared by all the three host genotypes in the basal zone (Fig. 4a), both of which belonged to unclassified Micrococcaceae, with higher relative abundance than most of the other niches (Fig. 4b). In the endosphere, three core ASVs, annotated as unclassified Burkholderiaceae,Rhizobacter, and Methylotenera (Fig. 4b) existed in both apical and basal segments of the EF genotype (Fig. 4a). The endosphere in the apical zone of LF2 genotype harboured the largest number of core ASVs, but these ASVs did not appear in other niches (Fig. 4a).

\section{Legume symbiotic rhizobia and total bacteria number}

The rhizotron experiment lasted for 15 days. As this was not long enough for chickpea plants to form visible nodules, an additional pot experiment was established using the same chickpea genotypes and soils. Nodules were collected and cleaned after 5 weeks growth. We performed $16 \mathrm{~S}$ rRNA gene amplicon shotgun sequencing 
of chickpea clean nodules and found ASV29417 consistently accounted for 90\%-95\% relative abundance in all the samples and considered it as the chickpea symbiotic rhizobia ASV (ASVcsr).

For the abundance of ASVcsr relative to the bulk soil, interactions between soil and other factors were not detected (Table 1). The enrichment of ASVcsr in the endosphere was greater than in the rhizosphere $(\mathrm{P}<$ $0.01,4.02$ vs $0.67 \log 2$ fold changes to bulk soil, Fig. 3c). In the rhizosphere of apical root, no variation of ASVcsr abundance among host genotypes was observed, while the EF genotype had more ASVcsr at the rhizosphere of basal zone than LF1 did. In the apical root endosphere, the genotype LF2 was colonised by the highest abundance of ASVcsr (82 vs 3.2-5.5 based on log2 FC to bulk soil), while this large genotypic difference was minimal in the basal zone. It should be noted that the ASVcsr was one of the core ASVs at the endosphere of LF2 apical roots, but not the core ASV in other niches or genotypes (Fig. 4b).

Bacterial number was estimated by the copy number of bacterial $16 \mathrm{~S}$ rRNA genes using qPCR. In the endosphere, there was a significant interaction between genotype and longitudinal root segment $(\mathrm{P}<0.01$, Table S2); the root tip of EF genotype was colonised by more bacteria than the LF genotypes (Fig. 3d), while genotypic variation in the basal zone was not observed. In contrast, bacterial number in the rhizosphere was only influenced by soil type $(\mathrm{P}<0.01$, Table S2 and Fig. S4).

\section{Root exudation}

To evaluate the possible mechanisms determining root-associated microbiomes via chickpea genetic variation and longitudinal niche differentiation, we analysed root exudation from apical and basal segments of chickpea genotypes with genetic variation in flowering time. To remove the microbial effect in degrading root exudates, sterilised soil (Soil-C) was used in the same rhizotron system and growing conditions as the microbiome experiment. Primary metabolites were analysed from the collected rhizosphere soil and adjusted by their concentrations in bulk soil. Based on the 34 detected root exudate compounds, unconstrained principal coordinate analyses (PCoA) and PERMANOVA using Bray-Curtis distance were performed, both of which demonstrated the considerable effect of longitudinal root segment on root exudate composition, indicated by high $\mathrm{R}^{2}$ value of $57 \%$ (Table S2). In the biplot, a separation was observed between samples of apical root and basal root, with the PCoA1 explaining $55 \%$ of the variation (Fig. 5a). In the apical root, the root exudates composition from the EF genotype differed greatly from the LF genotypes, while for the compounds from basal root, genotype LF2 differed from the other two genotypes.

The total amount of detected compounds from root exudation was greater in the root apical zone than the basal zone (Table S1). For the apical root, the EF genotype excreted more compounds than the LF genotypes; in particular, carboxylates from EF were $53 \%$ to $88 \%$ greater than those from the LF genotypes (Fig. 5b). There was no genotypic variation in rhizosphere soil $\mathrm{pH}$ (Table S2), and the rhizosphere soil at the apical zone had a lower $\mathrm{pH}$ than the basal zone (4.30 vs $4.85, \mathrm{P}<0.01$, Fig. $5 \mathrm{~d}$ ).

For every root exudate detected, we compared its abundance in the EF genotype with each of the LF genotypes, and selected the compounds with Log2 fold changes (FC) to both LF genotypes $>1$ and FDRadjusted $\mathrm{P}<0.01$ as EF-enriched, and $\log 2 \mathrm{FC}<-1$ and FDR-adjusted $\mathrm{P}<0.01$ as LF-enriched (Fig. 5c). No EF- or LF- enriched root exudates were found in the basal zone, while in the apical zone, propionic acid (carboxylate), benzoic acid (carboxylate), and phenylalanine (carboxylate) were identified as EF-enriched compounds, and succinic acid (carboxylate), mannose (sugar) and trehalose (sugar) were LF-enriched root exudates.

\section{Pore architecture of rhizosphere soil}

The soils used in the experiments had contrasting physical and chemical properties (Table S1). Hence, the contribution of rhizosphere soil architecture to the diversity of root microbiome was assessed using micro-CT scan. Firstly, we calculated soil porosity at the inner rhizosphere zone $(0-1 \mathrm{~mm}$ from root, Fig. 6a) relative to the outer rhizosphere zone (1-2 $\mathrm{mm}$ from root), and the microbiome Shannon index in the rhizosphere relative to the bulk soil for each of the phylal groups. The regression analysis using 5 soil types $\times 3$ chickpea genotypes identified that the correlation of rhizosphere soil porosity (based on micro-CT observed $>30 \mu \mathrm{m}$ 
diameter pores) was positive with Bacteroidetes diversity ( $\mathrm{P}<0.01$, Fig. 6b), but negative with Firmicutes diversity $(\mathrm{P}<0.05$, Fig. 6c). These significant correlations were only detected in the rhizosphere of the basal root.

\section{Discussion}

Our results highlight that niche differentiation along the longitudinal axis of a root system played a determinant role in structuring root-associated microbiota under the interactions with the factors of soil type, plant genotype, and rhizocompartment. The root microbiome of young seedlings with only 16 days growth reflects the genetic variation in flowering phenology of host genotypes. We describe a mechanism through which early flowering chickpea genotypes responded by establishing an endophyte community in the root tip with a greater diversity and higher compositional similarity to endophyte microbiome at the root basal zone. The host-niche selectivity of endophytes was associated with root exudation activities of primary metabolites. While the recruitment of rhizosphere microbiome across different soils was more conserved at the basal zone than root tip, the microbiome diversity in the basal rhizosphere was related to pore architecture of the rhizosphere soil, rather than plant root exudation.

Genetic variation in phenology of host plants characterised microbiota inhabiting the seedling root

Compared with the LF genotypes, the EF host was characterised by a distinct microbial community in the endosphere of the apical root. More importantly, the phenomenal assemblage of endophytes at EF genotype's root apex, and its differentiation from the LF genotypes were conserved, and consistent across diverse soil types. Our results demonstrate that the apical segment of the root system exerted a strong selection force on the soil microbiome, and the structured microbiota was potentially contributing to the plant's adaptation to the oncoming harsh environment, e.g. , promoting early maturity.

We have identified three important features of the endophytic microbiome established at the root apical zone of the EF host. Firstly, within the root system of the EF genotype, there was a homogeneous structure of the endosphere microbiome between the apical and basal zone, indicated by the high similarity of composition and diversity (Fig. 1b, c, 3b). In contrast, the apical and basal zones had divergent endophyte communities in the LF genotypes. Although the sampled apical fraction was only one day old in our experiment, the root apex of the EF genotype was able to rapidly select and assemble its endophyte microbiome with a similar composition to its 15-day old basal zone of the same root, which was presumably associated with its early maturity trait through this rapid development of an associated microbiome. Endophyte colonisation in the basal root tissues of the EF host was also more stable than in LF plants, as indicated by lower variation across soil types (Fig. 1d).

Secondly, the root of the EF genotype housed a greater diversity of the endophyte community in both the apical zone and basal zone, as indicated by a higher Shannon index than in LF plants (Fig. 3b). It has been determined that the seedling period in sorghum (Xu et al., 2018) and legumes (Moroenyane et al., 2020) was the critical stage in the life cycle to form the diversity of the root microbiome, as the diversity of root colonisers increased sharply during this stage, and then stabilised.

Thirdly, microbes closely colonising the root apex of the EF host served roles that potentially enhance plant growth. For example,Rhizobacter and Methylotenera microbes consistently enriched the apical zones of the EF genotype. Rhizobacter has been identified as a root endophytic bacteria in a diverse range of species (Pelletier et al., 2020), particularly in legumes (Hu et al., 2019; Brown et al., 2020), and was able to rescue growth inhibition induced by root-inhabiting fungi (Durán et al., 2018). The genusMethylotenera is a group of putative methylotrophic bacteria firmly interacting with plant growth as they are capable of utilizing one carbon source from the decomposition of the soil organic matter and the cell wall (Martin et al., 2020). Methylotenera can survive in low pH habitats (Kumar et al., 2019), hence might adapt to the apical zone of the DR genotype with more carboxylates secreted (as discussed below).

Root exudation activity in the apical zone of the EF host potentially contributed to forming 


\section{the features of its endophyte microbiome}

Root exudation activity in the apical zone of the EF host potentially contributed to forming the features of its endophyte microbiome. Compared with the LF genotypes, the EF host secreted a specific composition of metabolites from the apical zone, with more compounds of carboxylates and amino acids, especially, propionic acid, benzoic acid, and phenylalanine. The resulting acidic microhabitat can facilitate nutrient availability and mobilization for microbial utilisation, and stimulated the activity of particular microbial taxa, such as the enrichment of Burkholderia species in the low-pH rhizosphere of white lupin (Lupinus albus ) cluster roots (Weisskopf et al., 2011). Interestingly, one core ASV colonising apical root of EF genotype was also in Burkholderiaceae group (Fig. 4a).

The EF-enriched benzoic acid and phenylalanine observed in the present study are associated with biosynthesis of benzoxazinoids (BX) and salicylic acid (SA) in plant (Kudjordjie et al., 2019; de Vries et al., 2020). The effect of excreting BX and SA on the root microbiome have been thoroughly examined using genetic mutations (Lebeis et al., 2015; Cotton et al., 2019; Kudjordjie et al., 2019; Veach et al., 2019). A study on maize exhibited that the main role of BX-dependent metabolites was to stimulate methylophilic bacteria (Cotton et al., 2019). Notably, we also identified one endophytic core ASV belonging toMethylotenera genus in the apical zone of EF genotype (Fig. 4a), possibly due to the strong exudation of benzoic acid in the microsite.

\section{Deterministic processes of longitudinal niche differentiation shaped rhizosphere and endosphe- re microbiome}

Our five soils with different physical and chemical properties generated diverse communities of indigenous microbes. For the rhizospheric microbiome, soil type was the key driver influencing the composition and diversity, to a greater extent than other plant-associated attributors (Table 1, and Fig. 1a, d), which was in line with previous observations in legumes but using fewer soil types (Brown et al., 2020; Han et al., 2020). However, we also found that deterministic processes of longitudinal niche differentiation acted to select for certain rhizobacteria, e.g. two core ASVs (both belonging to Micrococcaceae) exceptionally enriched in the basal rhizosphere, independently of the host genotypes and soil types (Fig. 4). The rhizosphere microbiome in the root tip was variable and stochastic across different soils, possibly because the soil residence time of the sampled apical root was too short (only one day) to modulate its rhizosphere microbiome.

The recruitment of endophytic microbiota was governed by the host plant (including both genotype and its longitudinal niche effects), independent of the microbiome source (soil type). However, according to the sample-to-sample variation in the single soil, previous reports indicated that the assemblage of root microbiota was stochastic at root tips, and then stabilised in the mature sections (Thiergart et al., 2019). Nevertheless, our findings, based on diverse soils, demonstrated that endophyte colonisation was conserved at the root apex compared with the basal root, as indicated by the larger number of core ASVs (Fig.4 ) and higher compositional similarity among soil sources (Fig.1d ). Our observation was possibly attributed to the more active metabolism in the root tip of chickpea, especially secreting more carboxylates than at the basal zone (Fig. 5). Previous studies (Ross-Elliott et al., 2017; Canarini et al., 2019) revealed that the phloem (part of the endosphere) of the root tip was the location unloading most of the primary metabolites released by root, which potentially explains our finding that the recruitment effect of the root tip on endophyte acquisition exceeded the effect of soil type.

\section{Rhizosphere soil architecture determined rhizospheric microbiome acquisition}

Our results demonstrate that soil physical architecture surrounding the root zone were associated with the diversity of rhizosphere microbiome, but only at the basal zone of the root system. For example, by normalising the rhizosphere physical and microbial traits with bulk soil, it appears that increased rhizosphere porosity was correlated with greater diversity of microbes belonging to Bacteroidetes, but a reduced diversity of Firmicute microbes (Fig. 6 ). Modification of soil porosity by root elongation can alter microhabitats, through changes in gas and water flow (Young and Bengough, 2018). In chickpea plants, a more porous architecture of rhizosphere soil can favour water and gas permeability (Rabbi et al., 2018; Rabbi et al., 
2021). Firmicute bacteria might prefer the rhizosphere microsite with smaller pore volume and less oxygen, as Firmicutes included the Clostridia class which is a group of anaerobic bacteria (Teixeira et al., 2010). The enrichment and isolation under anaerobic conditions also confirmed a higher diversity of Firmicute isolates than Bacteroidetes (Malele et al., 2018).

\section{Symbiotic rhizobia acquisition was mainly in the endosphere of apical root}

The invasion process of chickpea symbiotic rhizobia (CSR) was the result of genotype-niche interactions, as we didn't detect any factors interacting with soil type on CSR abundance (Table 1). The spatial distribution of CSR showed more in the tissue of apical root than in the basal root (Fig. 3c). In the nodulation process, rhizobia in the soil may first colonise the root epidermis at the elongation zone following root-microbe signal communications or may directly bind to the zone of root hairs, while the exact sequence of this process is unknown (Poole et al., 2018). Our root apical zone included the root elongation zone, that was enriched in symbiotic rhizobia and likely facilitated the nodulation observed in the basal root sections.

In addition, CSR enrichment among chickpea genotypes was not related to the flowering phenological trait, as one LF genotype, rather than the EF genotype, harboured a greater proportion of CSR bacteria in root apex, and the enrichment was stable across soil types. Our results indicated the striking effect of host genotype in controlling nodulation processes, e.g., producing different host-specific flavonoids and root receptors that recognize symbiotic rhizobia (Sachs et al., 2018). CSR was not related to the phenological trait, e.g. , early maturity, while it might contribute to the amount of N fixed by chickpea (Sadras et al., 2016).

\section{Conclusions}

Overall, this study improved the resolution of root-associated niches harbouring microorganisms by identifying the importance of different segments along the longitudinal axis within one root system. Modulating the endophyte communities at the root tip by root exudation was associated with the genetic variation in phenology of the host plant. Across different soil types, the rhizosphere soil architecture potentially explained the diversity of microbial consortia at the basal root. Our findings demonstrate that longitudinal niche differentiation within a root architecture is an essential feature influencing the rhizobiome development and the microbial functions corresponding to plant growth.

\section{Acknowledgements}

Financial support of this work was provided by the Australian Research Council (project ID: IH140100013), the Grains Research and Development Corporation, the Department of Trade, Tourism and Investment of the South Australian Government, and The University of Adelaide. We acknowledge Ruey Toh and Jinjun Zhao for their assistance with growth chamber operations and sample analysis.

\section{Conflict of interest}

The authors declare that they have no conflict of interest

\section{Author contributions}

Yi Zhou and Matthew D Denton designed the study and interpreted all of the data. Yi Zhou conducted all the experiments. Yi Zhou and Matthew D Denton wrote the article. Sheikh Rabbi and Iain M Young conducted the micro-CT scanning to analyse the rhizosphere soil architecture and interpreted the results. David Fuentes, Andrew Merchant, and Brent N Kaiser undertook the root exudates analysis and interpreted the results. Jishun Li and Yanli Wei did the amplicon sequencing and interpreted the results. Yongle Li performed the genomic analysis of chickpea genotypes and interpreted the results. All authors contributed to revising the manuscript, and approved the final submission.

\section{Data availability statement}

The raw sequenced data from the Illumina MiSeq platform are available at National Center for Biotechnology Information (NCBI), Sequence Read Archive (https://www.ncbi.nlm.nih.gov/sra) with the accession number 
PRJNA595445. All relevant data and codes supporting the findings of this study are available from the corresponding author upon request.

\section{References}

Abbo, S., Lev-Yadun, S., and Galwey, N. (2002). Vernalization response of wild chickpea. New Phytologist , 154, 695-701.

Baluška, F., Mancuso, S., Volkmann, D., and Barlow, P.W. (2010). Root apex transition zone: a signallingresponse nexus in the root. Trends in Plant Science, 15, 402-408.

Benizri, E., Baudoin, E., and Guckert, A. (2001). Root colonization by inoculated plant growth-promoting rhizobacteria. Biocontrol Science and Technology, 11, 557-574.

Bolyen, E., Rideout, J.R., Dillon, M.R., Bokulich, N.A., Abnet, C.C., Al-Ghalith, G.A. et al. (2019). Reproducible, interactive, scalable and extensible microbiome data science using QIIME 2. Nature Biotechnology , 37, 852-857.

Brown, S.P., Grillo, M.A., Podowski, J.C., and Heath, K.D. (2020). Soil origin and plant genotype structure distinct microbiome compartments in the model legume Medicago truncatula . Microbiome, 8.

Bulgarelli, D., Rott, M., Schlaeppi, K., van Themaat, E.V.L., Ahmadinejad, N., Assenza, F. et al. (2012). Revealing structure and assembly cues for Arabidopsis root-inhabiting bacterial microbiota. Nature , 488, 91-95.

Callahan, B.J., McMurdie, P.J., Rosen, M.J., Han, A.W., Johnson, A.J.A., and Holmes, S.P. (2016). DADA2: high-resolution sample inference from Illumina amplicon data.Nature Methods , 13, 581-583.

Canarini, A., Kaiser, C., Merchant, A., Richter, A., and Wanek, W. (2019). Root exudation of primary metabolites: mechanisms and their roles in plant responses to environmental stimuli. Frontiers in Plant Science, 10, 157.

Conselvan, G.B., Fuentes, D., Merchant, A., Peggion, C., Francioso, O., and Carletti, P. (2018). Effects of humic substances and indole-3-acetic acid on Arabidopsis sugar and amino acid metabolic profile. Plant and Soil , 426, 1-16.

Cotton, T.E.A., Petriacq, P., Cameron, D.D., Al Meselmani, M., Schwarzenbacher, R., Rolfe, S.A., and Ton, J. (2019). Metabolic regulation of the maize rhizobiome by benzoxazinoids. Isme Journal , 13, 1647-1658.

Darwin, C. (1897). The power of movement in plants . London: John Murray.

de Vries, F.T., Griffiths, R.I., Knight, C.G., Nicolitch, O., and Williams, A. (2020). Harnessing rhizosphere microbiomes for drought-resilient crop production.Science , 368, 270-274.

DeAngelis, K.M., Brodie, E.L., DeSantis, T.Z., Andersen, G.L., Lindow, S.E., and Firestone, M.K. (2009). Selective progressive response of soil microbial community to wild oat roots. Isme Journal , 3, 168-178.

Dombrowski, N., Schlaeppi, K., Agler, M.T., Hacquard, S., Kemen, E., Garrido-Oter, R. et al. (2017). Root microbiota dynamics of perennial Arabis alpina are dependent on soil residence time but independent of flowering time. Isme Journal , 11, 43-55.

Durán, P., Thiergart, T., Garrido-Oter, R., Agler, M., Kemen, E., Schulze-Lefert, P., and Hacquard, S. (2018). Microbial interkingdom interactions in roots promote Arabidopsis survival. Cell , 175, 973-983.

Edwards, J., Johnson, C., Santos-Medellin, C., Lurie, E., Podishetty, N.K., Bhatnagar, S. et al. (2015). Structure, variation, and assembly of the root-associated microbiomes of rice. Proceedings of the National Academy of Sciences of the United States of America, 112, E911-E920.

Edwards, J.A., Santos-Medellín, C.M., Liechty, Z.S., Nguyen, B., Lurie, E., Eason, S. et al. (2018). Compositional shifts in root-associated bacterial and archaeal microbiota track the plant life cycle in field-grown 
rice. PLoS Biology , 16, e2003862.

Flavel, R.J., Guppy, C.N., Rabbi, S.M., and Young, I.M. (2017). An image processing and analysis tool for identifying and analysing complex plant root systems in 3D soil using non-destructive analysis: Root1. PloS One , 12, e0176433.

Greenlon, A., Chang, P.L., Damtew, Z.M., Muleta, A., Carrasquilla-Garcia, N., Kim, D. et al. (2019). Globallevel population genomics reveals differential effects of geography and phylogeny on horizontal gene transfer in soil bacteria.Proceedings of the National Academy of Sciences , 116, 15200-15209.

Han, Q., Ma, Q., Chen, Y., Tian, B., Xu, L.X., Bai, Y. et al. (2020). Variation in rhizosphere microbial communities and its association with the symbiotic efficiency of rhizobia in soybean. Isme Journal , 14, 1915-1928.

Hsieh, T., Ma, K., and Chao, A. (2016). iNEXT: an R package for rarefaction and extrapolation of species diversity (Hill numbers). Methods in Ecology and Evolution , 7, 1451-1456.

Hu, W., Strom, N.B., Haarith, D., Chen, S., and Bushley, K.E. (2019). Seasonal variation and crop sequences shape the structure of bacterial communities in cysts of soybean cyst nematode. Frontiers in Microbiology , $10,2671$.

Kaloki, P., Luo, Q., Trethowan, R., and Tan, D.K. (2019). Can the development of drought tolerant ideotype sustain Australian chickpea yield? International journal of biometeorology , 63, 393-403.

Kawasaki, A., Donn, S., Ryan, P.R., Mathesius, U., Devilla, R., Jones, A., and Watt, M. (2016). Microbiome and exudates of the root and rhizosphere of brachypodium distachyon, a model for wheat. PloS One , 11, e0164533.

Kawasaki, A., Dennis, P.G., Forstner, C., Raghavendra, A.K., Richardson, A.E., Watt, M. et al. (2021a). The microbiomes on the roots of wheat (Triticum aestivum L.) and rice (Oryza sativa L.) exhibit significant differences in structure between root types and along root axes. Functional Plant Biology .

Kawasaki, A., Dennis, P.G., Forstner, C., Raghavendra, A.K., Mathesius, U., Richardson, A.E. et al. (2021b). Manipulating exudate composition from root apices shapes the microbiome throughout the root system. Plant Physiology .

Kudjordjie, E.N., Sapkota, R., Steffensen, S.K., Fomsgaard, I.S., and Nicolaisen, M. (2019). Maize synthesized benzoxazinoids affect the host associated microbiome.Microbiome , 7 .

Kumar, M., Kour, D., Yadav, A.N., Saxena, R., Rai, P.K., Jyoti, A., and Tomar, R.S. (2019) Biodiversity of methylotrophic microbial communities and their potential role in mitigation of abiotic stresses in plants. Biologia , 74, 287-308.

Lebeis, S.L., Paredes, S.H., Lundberg, D.S., Breakfield, N., Gehring, J., McDonald, M. et al. (2015). Salicylic acid modulates colonization of the root microbiome by specific bacterial taxa. Science, 349, 860-864.

Li, Y., Ruperao, P., Batley, J., Edwards, D., Davidson, J., Hobson, K., and Sutton, T. (2017). Genome analysis identified novel candidate genes for ascochyta blight resistance in chickpea using whole genome re-sequencing data.Frontiers in Plant Science, 8, 359.

Lu, T., Ke, M.J., Lavoie, M., Jin, Y.J., Fan, X.J., Zhang, Z.Y. et al. (2018). Rhizosphere microorganisms can influence the timing of plant flowering. Microbiome , 6, 1.

Malele, I., Nyingilili, H., Lyaruu, E., Tauzin, M., Ollivier, B.B., Cayol, J.-L. et al. (2018). Bacterial diversity obtained by culturable approaches in the gut of Glossina pallidipes population from a non sleeping sickness focus in Tanzania: preliminary results. BMC Microbiology , 18, 107-116.

Martin, B.C., Alarcon, M.S., Gleeson, D., Middleton, J.A., Fraser, M.W., Ryan, M.H. et al. (2020). Root microbiomes as indicators of seagrass health. FEMS Microbiology Ecology , 96. 
Moroenyane, I., Tremblay, J., and Yergeau, É. (2020). Temporal and spatial interactions modulate the soybean microbiome. FEMS Microbiology Ecology, 97, 1.

Oksanen, J., Kindt, R., Legendre, P., O'Hara, B., Stevens, M.H.H., Oksanen, M.J., and Suggests, M. (2007). The vegan package. Community Ecology Package, 10, 631-637.

Parks, D.H., Tyson, G.W., Hugenholtz, P., and Beiko, R.G. (2014). STAMP: statistical analysis of taxonomic and functional profiles. Bioinformatics , 30, 3123-3124.

Pelletier, D.A., Li, Z., Lu, T.-Y.S., Zhang, L., Hu, Z., Morris, G.P. et al. (2020). Genome sequences of 42 bacteria isolated from sorghum bicolor roots. Microbiology Resource Announcements , 9.

Poole, P., Ramachandran, V., and Terpolilli, J. (2018). Rhizobia: from saprophytes to endosymbionts. Nature Reviews Microbiology, 16, 291.

Quast, C., Pruesse, E., Yilmaz, P., Gerken, J., Schweer, T., Yarza, P. et al. (2012). The SILVA ribosomal RNA gene database project: improved data processing and web-based tools. Nucleic acids research , 41, D590-D596.

Rabbi, S.M., Tighe, M.K., Flavel, R.J., Kaiser, B.N., Guppy, C.N., Zhang, X., and Young, I.M. (2018). Plant roots redesign the rhizosphere to alter the three-dimensional physical architecture and water dynamics. New Phytologist, 219, 542-550.

Rabbi, S.M., Tighe, M.K., Warren, C.R., Zhou, Y., Denton, M.D., Barbour, M.M., and Young, I.M. (2021). High water availability in drought tolerant crops is driven by root engineering of the soil micro-habitat. Geoderma , 383, 114738.

Rayment, G.E., and Lyons, D.J. (2011)Soil chemical methods: Australasia : CSIRO publishing.

Reinhold-Hurek, B., and Hurek, T. (1998). Life in grasses: diazotrophic endophytes. Trends in Microbiology , 6, 139-144.

Ridge, S., Deokar, A., Lee, R., Daba, K., Macknight, R.C., Weller, J.L., and Tar'an, B. (2017). The chickpea Early Flowering 1 (Ef1) locus is an ortholog of Arabidopsis ELF3.Plant physiology, 175, 802-815.

Robinson, M.D., McCarthy, D.J., and Smyth, G.K. (2010). edgeR: a Bioconductor package for differential expression analysis of digital gene expression data.Bioinformatics , 26, 139-140.

Ross-Elliott, T.J., Jensen, K.H., Haaning, K.S., Wager, B.M., Knoblauch, J., Howell, A.H. et al. (2017). Phloem unloading in Arabidopsis roots is convective and regulated by the phloem-pole pericycle. Elife, 6, e24125.

Ruger, L., Feng, K., Dumack, K., Freudenthal, J., Chen, Y., Sun, R. et al. (2021) Assembly patterns of the rhizosphere microbiome along the longitudinal root axis of maize (Zea mays L.). Frontiers in Microbiology , 12, 237.

Sachs, J.L., Quides, K.W., and Wendlandt, C.E. (2018). Legumes versus rhizobia: a model for ongoing conflict in symbiosis. New Phytologist , 219, 1199-1206.

Sadras, V.O., Lake, L., Li, Y., Farquharson, E.A., and Sutton, T. (2016). Phenotypic plasticity and its genetic regulation for yield, nitrogen fixation and $\delta 13 \mathrm{C}$ in chickpea crops under varying water regimes. Journal of Experimental Botany , 67, 4339-4351.

Sasse, J., Martinoia, E., and Northen, T. (2017). Feed your friends: do plant exudates shape the root microbiome? Trends in Plant Science, 25-41.

Schindelin, J., Arganda-Carreras, I., Frise, E., Kaynig, V., Longair, M., Pietzsch, T. et al. (2012). Fiji: an open-source platform for biological-image analysis. Nature Methods , 9, 676. 
Teixeira, L.C., Peixoto, R.S., Cury, J.C., Sul, W.J., Pellizari, V.H., Tiedje, J., and Rosado, A.S. (2010). Bacterial diversity in rhizosphere soil from Antarctic vascular plants of Admiralty Bay, maritime Antarctica. Isme Journal , 4, 989-1001.

Thiergart, T., Zgadzaj, R., Bozsoki, Z., Garrido-Oter, R., Radutoiu, S., and Schulze-Lefert, P. (2019). Lotus japonicus symbiosis genes impact microbial interactions between symbionts and multikingdom commensal communities. Mbio, 10.

Veach, A.M., Morris, R., Yip, D.Z., Yang, Z.K., Engle, N.L., Cregger, M.A. et al. (2019). Rhizosphere microbiomes diverge among Populus trichocarpa plant-host genotypes and chemotypes, but it depends on soil origin. Microbiome, 7 .

Von Wettberg, E.J., Chang, P.L., Başdemir, F., Carrasquila-Garcia, N., Korbu, L.B., Moenga, S.M. et al. (2018). Ecology and genomics of an important crop wild relative as a prelude to agricultural innovation. Nature Communications , 9, 1-13.

Wang, X., Tang, C., Severi, J., Butterly, C.R., and Baldock, J.A. (2016). Rhizosphere priming effect on soil organic carbon decomposition under plant species differing in soil acidification and root exudation. New Phytologist , 211, 864-873.

Weisskopf, L., Heller, S., and Eberl, L. (2011). Burkholderia species are major inhabitants of white lupin cluster roots. Applied and Environmental Microbiology , 77, 7715-7720.

Wouterlood, M., Cawthray, G.R., Scanlon, T.T., Lambers, H., and Veneklaas, E.J. (2004). Carboxylate concentrations in the rhizosphere of lateral roots of chickpea (Cicer arietinum) increase during plant development, but are not correlated with phosphorus status of soil or plants. New Phytologist, 162, 745-753.

Xu, L., Naylor, D., Dong, Z.B., Simmons, T., Pierroz, G., Hixson, K.K. et al. (2018). Drought delays development of the sorghum root microbiome and enriches for monoderm bacteria. Proceedings of the National Academy of Sciences of the United States of America, 115, E4284-E4293.

Young, I., and Bengough, A. (2018). The search for the meaning of life in soil: an opinion. European Journal of Soil Science, 69, 31-38.

Zhou, Y., Coventry, D.R., Gupta, V.V., Fuentes, D., Merchant, A., Kaiser, B.N. et al. (2020). The preceding root system drives the composition and function of the rhizosphere microbiome. Genome Biology, 21, 1-19.

\section{Figure Legends}

Fig. 1. The effect of chickpea genotype, soil type, and root segment on microbial composition in the rhizosphere and endosphere.

Non-metric multidimensional scaling (NMDS) analysis based on Bray-Curtis distance was conducted to show the microbiome composition in (a) bulk soil and rhizosphere, and (b) endosphere. Bray-Curtis distance (c) between apical and basal zone of the root, and (d) between soils were calculated in the rhizosphere and endosphere. One early flowering (EF) and two late flowering (LF1 and LF2) chickpea genotypes were studied. The same lowercase letters indicated no significant differences $(\mathrm{P}<0.05)$ between genotypes based on Tukey HSD post-hoc pairwise comparison test.

Fig. 2. Taxonomic composition of microbiome at phyla level across different soils, chickpea genotypes, rhizocompartments (bulk soil, rhizosphere, and endosphere), and root segments (apical and basal zones). One early flowering (EF) and two late flowering (LF1 and LF2) chickpea genotypes were studied.

Fig. 3. The diversity of microbial community, and abundance of microbial population able to inoculate chickpea plant.

The experiment studied rhizosphere and endosphere microbiome at apical and basal roots of chickpea genotypes growing in five different soils. The presented data included Shannon index of (a) rhizosphere and 
(b) endosphere microbiome, (c) abundance of chickpea symbiotic rhizobia ASV relative to bulk soil, and (d) endospheric bacteria number estimated by the copy number of bacterial 16S rRNA gene. One early flowering (EF) and two late flowering (LF1 and LF2) chickpea genotypes were evaluated. The same lowercase letters indicated no significant differences $(\mathrm{P}<0.05)$ between genotypes based on Tukey HSD post-hoc pairwise comparison test.

Fig. 4. Comparison of core microbes at root-associated niches of chickpea genotypes across different soils

In each niche, the ASVs with relative abundance $>90 \%$ of the most abundant ASV were defined as dominant ASVs (dASVs). dASVs appearing in all five soils were assumed to be core microbes. (a) the number of core microbes in each niche and (b) the heatmap showing the relative abundance of core microbes. * indicated the core microbes. One early flowering (EF) and two late flowering (LF1 and LF2) chickpea genotypes were evaluated.

Fig. 5. Root exudation at the apical and basal root zones of chickpea genotypes with contrasting drought resistance.

One early flowering (EF) and two late flowering (LF1 and LF2) chickpea genotypes were evaluated. (a) principal coordinate analysis $(\mathrm{PCoA})$ was based on the abundance of 34 detected root exudates and BrayCurtis distance between samples. (b) three chemical groups of root exudates influenced by chickpea genotypes and root zones. (c) the root exudation compounds with Log2 fold changes of EF/LF $>1$ or $<-1$. (d) rhizosphere soil $\mathrm{pH}$ at apical and basal zones of chickpea genotypes. The same lowercase letters (uppercase letter for the total root exudates) indicated no significant differences $(\mathrm{P}<0.05)$ between genotypes based on Tukey HSD post-hoc pairwise comparison test. * indicated the FDR-adjusted $\mathrm{P}<0.01$.

Fig. 6. Soil architecture associated with the diversity of microbial consortia in rhizosphere

Rhizosphere soil porosity change ratio indicated by (a) the soil porosity at $0-1 \mathrm{~mm}$ relative to $1-2 \mathrm{~mm}$ root zone, and (b) its correlation with the diversity of microbes belonging to Bacteroidetes and Firmicutes at the rhizosphere of basal roots. Presented data included five soil types and three chickpea genotypes. ${ }^{*}$ and ${ }^{* *}$ indicated the significant correlation at $\mathrm{P}<0.05$ and $\mathrm{P}<0.01$, respectively.

\section{Hosted file}

Figures.docx available at https://authorea.com/users/351725/articles/557180-geneticvariation-in-host-plant-phenology-affects-microbial-assemblages-at-longitudinal-nichesof-the-seedling-root

\section{Hosted file}

Tables. docx available at https://authorea.com/users/351725/articles/557180-genetic-variationin-host-plant-phenology-affects-microbial-assemblages-at-longitudinal-niches-of-theseedling-root 